\title{
Design and Assessment of a Task-Driven Introductory Data Science Course Taught Concurrently in Multiple Languages: Python, R, and MATLAB
}

\author{
Ting Xiao \\ University of North Texas \\ Denton, Texas, USA \\ ting.xiao@unt.edu
}

\author{
Ronald I. Greenberg \\ Loyola University Chicago \\ Chicago, Illinois, USA \\ rig@cs.luc.edu
}

\author{
Mark V. Albert \\ University of North Texas \\ Denton, Texas, USA \\ mark.albert@unt.edu
}

\begin{abstract}
We discuss the design, effectiveness, and curricular impacts of an introductory data science course that uniquely meets a number of competing constraints. Student needs range from an initial exploration for future data scientists to the only computing course for some science students. Initially, associated research labs, departments, and industries provided differing perspectives on skills and preferred programming languages. The focus was on practical programming skills and learning through demos to develop useful skills quickly. The task-driven nature also enabled the mutual development of resources for each of the top three requested languages, Python, R, and MATLAB, allowing students to concurrently complete the course in any of the three languages. Tutorials in all three languages were produced to prepare students for the language-independent task-driven assignments given each day. For consistency all tutorials and assignments use the Jupyter notebook cell-based workflow. Feedback and performance measures of three student cohorts (Spring 2018, Fall 2018, and Spring 2019) are analyzed. Feedback included an average rating that met or exceeded 4 ("substantial progress") out of 5 for each separate cohort on all four general educational objectives (Basic Understanding, Developing Skills, Application, and Numeric Methods). $75 \%$ of responding students indicated a preference for the multi-language course design. The course also attracted a majority of women every semester $(63 \%$ female, average). Because of these benefits, this course became the recommended first programming course for a newly developed and approved undergraduate data science major. All material is online and available at https://sites.google.com/view/comp-180
\end{abstract}

\section{CCS CONCEPTS}

- Applied Computing $\rightarrow$ Education; $•$ Mathematics of Computing $\rightarrow$ Statistical Paradigms; $\bullet$ Computing Methodologies $\rightarrow$ Learning Paradigms; Machine Learning.

\section{KEYWORDS}

Data science; computational science; task-driven; multiple languages; Jupyter notebook

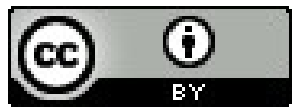

This work is licensed under a Creative Commons Attribution International 4.0 License. ITiCSE 2021, fune 26-fuly 1, 2021, Virtual Event, Germany.

(C) 2021 Copyright held by the owner/author(s).

ACM ISBN 978-1-4503-8214-4/21/06.

https://doi.org/10.1145/3430665.3456364
ACM Reference Format:

Ting Xiao, Ronald I. Greenberg, and Mark V. Albert. 2021. Design and Assessment of a Task-Driven Introductory Data Science Course Taught Concurrently in Multiple Languages: Python, R, and MATLAB. In Proceedings of the 2021 ACM Conference on Innovation and Technology in Computer Science Education (ITiCSE '21), June 26-fuly 1, 2021, Virtual Event, Germany. ACM, New York, NY, USA, 6 pages. https://doi.org/10.1145/3430665.3456364

\section{INTRODUCTION}

The amount of data generated, processed, and analyzed is increasing at an exceptional rate. Statisticians and computer scientists have been critical to meet the industry demands to leverage this data for actionable insights in business, health, and education. Available information technology approaches permit more robust, automated data-driven decision making by collecting massive data sets [16]. Over time, the tools to manage these data processing tasks have become more standardized and accessible across fields. Because of this, there is an increased expectation that scientists not only understand their domain, but also have a working understanding of applied computer science and statistics to achieve their aims [10].

Training scientists for our data-driven world also has inherent overlap with a field of explosive growth in industry; Data science is valued across academia and industry due to it its ability to extract meaningful insights from data using a combination of domain knowledge, programming skills, and statistics [13]. With the right data infrastructure, a data scientist has the ability to take complex, unstructured sources of data and communicate relevant findings to CEOs, managers, or even clients through automated systems. For this reason, data science is one of the most attractive career paths. Glassdoor [8] has ranked data scientist in the top three and usually as the \#1 Best Job in America since at least 2016, in part due to high salary and increasing demand in multiple projections [17]. LinkedIn [12] listed data scientist as the most promising job of 2019, along with multiple data-science-related skills as the most in-demand by companies. In short, there is a significant demand to not only provide working scientists with introductory data science skills, but also to prepare future data scientists.

Given the teaching resource constraints that computing departments and science departments face, we discuss a course design that offers a creative solution to teaching both science students in a potentially terminal computing course as well as beginning undergraduate data science majors. No previous programming experience is required for science students, and only data science students with little or no programming experience are encouraged to begin with this course. For beginning data science students, this 
provides appropriate context and scaffolding for their future course selections and career paths.

One of the consistent challenges in designing a computer science or data science curriculum is choosing a programming language for the introductory course sequence [3, 5, 11]. In our particular case, we had competing interests: psychology and engineering stakeholders suggested MATLAB, statisticians suggested R, while Python is most popular, especially for computer science [19]. This was addressed by designing course materials to provide code-based instruction in all three languages, though the exercises and evaluations are language agnostic. In practice, we found the instructor did not need to be well versed in all three languages offered, since the tutorials provide sufficient context for instructors fluent with at least one of the programming languages, and this is all at an introductory level.

Due to the nature of instruction in multiple languages, assignments and quizzes are necessarily task-driven rather than tooldriven. In contrast to the practice in other introductory computer science courses, theoretical concepts and computing foundations that are useful to computer science students come secondary to more immediate, practical concerns in working with data. Also, since the course is likely to be a terminal computing course for many, students are pushed to be self-sufficient in basic data analysis tasks. Daily instructional efforts are task-oriented based on what is most useful to students. The data analysis tutorials for each language employ a cell-based run/display-results cycle using Jupyter Notebooks for consistency in instruction. Notably, all efforts in the course are performed directly on the computer and motivated by externally driven data analysis tasks emphasizing real-world applications.

This report is aimed at computing department curriculum administrators who would like to consider this course design approach, particularly in reaching out to provide instruction better tailored for science departments than most object-oriented programming (OOP) traditional computing introductions. Also, instructors of related courses can directly benefit, since all the materials are readily available online for instructors in any of the three languages mentioned.

The course was originally designed to appeal to physical and social science students, but through feedback and experience with computer science and statistics students, the target audience was expanded to include early data science undergraduates. Most science undergraduates were not well-suited for the object-oriented, Java-based CS1 course mainly intended for undergraduate computer science students. Such students also were underserved by an alternative python-based "Introduction to Computing" course that does not emphasize data analysis, in part due to its role as a University Core course serving students across all disciplines. After a few semesters of instruction it was clear that the course also appealed to more advanced science students who took the course as an elective. During curricular development of a new data science undergraduate major, this course was chosen as the introductory programming course for students new to the major as it provided a beneficial foundation for future work.

This approach to course development, and the data science program in which it was embedded, are further supported by observations in similar data science courses and programs. The course described here was embedded in a data science degree program at Loyola University Chicago run jointly by the Department of Computer Science and the Department of Mathematics \& Statistics; the equal participation by these two departments was helpful for ensuring a good balance of computing and statistics [2]. Similar course designs can serve a purpose as part of a developing data science degree program in a larger university setting [14] or a smaller liberal-arts college with limited computing resources [15]. Since programming experience is not required as a prerequisite to this course, it can serve as an on-ramp to data science for non-CS students $[6,7,18]$ or as a data-centric second course in computing [4]. The course content below addresses a number of competencies for a data science curriculum [1]. We demonstrate that this data-centric approach better engages and supports a diversity of students [9].

\section{COURSE CONTENT}

This course aims to teach data analysis and visualization in an applied scientific context - analyzing data quickly for understanding by the individual scientist, sharing automated workflows with collaborators, and preparing results for later publication. This course emphasizes rapid, interactive, and reproducible collaborative analysis of data for scientific contribution. At the end of this course, students are to be well versed in the use of an interactive environment for data analysis (Python, R, or MATLAB), with an ability to manage the collection of data, create automated processes for analysis, use collaborative tools, and rapidly report quantitative findings.

The content is broken down in the outline below, with associated assignments numbered. There are tutorials for each week of the course spanning the necessary content to complete the assignments.

Data visualization (3 weeks):

1: Basic Language Syntax

2: Simple charts (pie charts, bar charts)

3: Grouped bar plots, basic language syntax

4: Histograms, proofing images for publication

5: 2D plots (line plots, scatter plots, subplots)

6: 3D plots (contour maps, surface plots)

Data structures, including data frames (2 weeks)

7: Primer on data structures

8: Applications of data structures

9: Primer on data frames

10: Applications of data frames

Image processing classification demo (2 weeks)

11: Simple image processing

12: Simple image classification demo

13: k-Nearest Neighbors classification

Regression demo (1 week)

14: Polynomial regression

Project group work and presentations (3 weeks)

Project proposals, in class updates, presentations, final reports

Advanced topics and applications, examples (1 week)

Wearables in health care,

Discovering particles using data science

Online automated predictive modeling competition (2 weeks)

Kaggle analytics competition

Review exercises 
We will step through reasoning and insights from some of the curricular choices. The initial focus on data visualization provides a more appealing demo-driven introduction to the course before the necessary survey of data structures afterwards. Data frames are emphasized, since 2D tabular data structures are quicker for students to adopt on the time scale of this course. The image processing task is to classify images based on color histograms, first by hand, using if/then statements, and then later seeing the way in which k-nearest neighbors performs the same task. The first full lecture in the course is for exposure to the concepts and practice of machine learning. Project selection is generally biased toward classification or regression to give more first-hand experience after initial, relatively short exposure. After project work, advanced data analysis topics are presentated by the instructor or colleagues. Finally, the last two weeks are reserved for a class-wide automated predictive modeling competition in the style of Kaggle.com with real-time leaderboards and scoring of models. During the competition phase, exercises are given to recap material in the course in a format similar to what will be seen on the final exam.

\section{CLASSROOM APPROACH}

As an application-oriented course, reading materials and theory are minimal. Code-based tutorials are given daily with languageagnostic assignments providing much of the content and expectations in the course. Students are encouraged to use search engines regularly and are given tips and techniques throughout to help them solve problems efficiently.

The instructor regularly emphasized a single language for brief, daily code walks to help complete assignments, generally the first 5-10 minutes of each class. There are daily data analysis tasks given as assignments. Weekly full-session quizzes (Wednesday in an MWF class schedule) were given in lieu of a mix of quizzes and exams. Such quizzes were timed on the computer with online submission and resemble assignments in nature, practical rather than theoretical. This approach stresses the need to not just complete assignments but learn from them to become more efficient.

Students beginning the course are given the context in which these skills are applied and encouraged early on to select one of the three data analysis languages to specialize in based on their needs and goals. Students are free to switch languages at will, but the need for consistency is stressed if this is their first exposure to programming. Enough information is provided in the language-specific tutorials to complete the tasks with minimal external resources, however, some searching online is instructed, and later expected, to solve assignments.

\subsection{Assignments and Projects}

Assignments were designed to motivate students to synthesize solutions from the tutorials and tailor their computing environment to suit their needs. With the emphasis of practice over principle, assignments generally advance the concepts in the course naturally based on need, and evaluations are more oriented towards gauging ability to apply what is learned in the field.

In initial iterations of the course, assignments were due within one week after they were assigned (e.g., Monday assignments due on Sunday; Friday assignments due the next Thursday), however, students would regularly focus on the previous assignment when new assignments were given, so later iterations required assignments to be due before the end of class in the next class session. This motivated preparation at home but allowed for in-class questions and trouble-shooting that may have been necessary for struggling students.

Results had to be presentable, with appropriate comments for someone to follow what has been done. Assignments were turned in individually, though students were encouraged to discuss anything freely and openly that is not a quiz or exam, including reading each other's code, but they were not allowed to copy and paste or any thoughtless equivalent. They were told to ask if in doubt and to at least document their source clearly and explicitly. Notably, assignments were given on an almost daily basis. With appropriate preparation and attention, most could be accomplished in class giving some time for experimentation. To encourage in-class efforts and to provide precise feedback, students were encouraged to show assignments to the instructor or the TA in class, before submitting to the course management software, to guarantee full points. Emphasis was placed on keeping up to date in the course as assignments were frequent and quizzes were weekly.

Attendance was not required except on quiz and exam days. There was no direct participation grading, but students were made aware that there has been a strong correlation between engagement and accomplishment in courses, especially for those that are struggling with the material. Because the weekly quizzes and final exam constituted 3-4 times more points than the assignments, this motivated students to come to class and perform assignments on their own without formally requiring regular, direct supervision.

A few weeks into the course students selected among a small number of collaborative projects. This began with a task for individual one-paragraph descriptions of desired projects which, after classification and regression is introduced, are often sufficiently complex for a multi-week project effort. Students were encouraged to reach out to people who may have data that could be analyzed on their behalf. Groups were 2-3 people, with individuals working alone strictly not allowed, in order to encourage collaboration. After the projects were selected, progress was encouraged and tracked through initial group project proposals, in class oral updates, project presentations, and short, written final reports with the intention to provide a showcase for their efforts.

\subsection{Assessment Strategy}

Students were evaluated in class on a weekly basis in a manner similar to assignments - data analysis tasks using a computer, but under time pressure and without help from others. Students were strongly encouraged to attempt to solve the tasks iteratively and incrementally - writing code that works first, but works poorly, and improving from there, rather than writing perfect code from beginning to end. When finished with a quiz or an exam, students were also encouraged to use the extra time to organize their materials to become more efficient at data analysis tasks similar to the assignments. All quizzes were due at the end of the class, with an analysis notebook (.ipynb file) submitted to the course management system. 
Table 1: Majors present in the classes. If multiple majors were declared, they are counted as appropriate fractions.

\begin{tabular}{lc|ccc}
\hline Major & Total & S18 & F18 & S19 \\
\hline Neuroscience & 14.53 & 4.53 & 2.75 & 7.25 \\
Psychology & 6.16 & 0.83 & 3.58 & 1.75 \\
CS/Math/Stat & 10.16 & 4.66 & 3.5 & 2 \\
Business/Economics & 4 & 0 & 2 & 2 \\
Misc & 17.14 & 5.97 & 4.17 & 7 \\
\hline
\end{tabular}

In line with a practical emphasis, students were encouraged to bring their own laptops and configure the system appropriately to complete the assignments, though machines were available to students on-site as well. Students were expected to use the same machine for quizzes and exams as for assignments. Students were asked to anticipate potential failures in data analysis due to hardware or software issues (e.g. not enough battery, OS freezes without saving, etc.), however, they were allowed to drop at most one quiz because of such an issue. Since students were observed during exams, and lab computers were provided for many, this policy was only used one time in three semesters.

\section{COURSE EXPERIENCE AND FEEDBACK}

The course was titled "COMP 180: Computing and Data Analysis for the Sciences". It was taught by the first author at her prior institution over three semesters from Spring 2018 to Spring 2019 with a total of 52 students. Also, undergraduate students in the course varied in terms of majors and academic status, providing a number of diverse perspectives.

\subsection{Student Composition}

The initial design of the course was as a terminal computing course for students that were not intending to complete the course progression for a computer science major or minor. Students in psychology and neuroscience were told this course could serve as one of their quantitative electives, hence the large representation of these majors. A few students finished the course to solidify their data analysis skills even though it did not serve a role in their graduation requirements; based on this feedback, the curricular design evolved to encourage interest in pursuing data science as well. The undergraduate data science major was approved to be effective after the last of these three semesters, so we are not able to report on enrollment and feedback of first-year data science students. However, over 10 computer science, math, and statistics students provided their feedback which supported this curricular adjustment.

The distribution of majors in the course represents the role as a course for science students without traditionally extensive computing foundations, predominantly neuroscience and psychology (Table 1). The distribution of student years was skewed toward junior and senior students by credit hours, although students were present from all four years of undergraduate study (Table 2). It is worth noting that this course had a majority of women in each cohort with $63.5 \%$ female representation on average (Table 3 ).
Table 2: Academic year of students by credit hours

\begin{tabular}{lc|ccc}
\hline Year & Total & S18 & F18 & S19 \\
\hline Freshman & 6 & 3 & 1 & 2 \\
Sophomore & 9 & 1 & 2 & 6 \\
Junior & 18 & 4 & 8 & 6 \\
Senior & 19 & 8 & 5 & 6 \\
\hline
\end{tabular}

Table 3: Female to male class composition

\begin{tabular}{lc|ccc}
\hline Sex & Total & S18 & F18 & S19 \\
\hline Female & 33 & 11 & 9 & 13 \\
Male & 19 & 5 & 7 & 7 \\
\hline$\%$ Female & $64 \%$ & $69 \%$ & $56 \%$ & $65 \%$ \\
\hline
\end{tabular}

Table 4: Quantitative student feedback on learning of relevant objectives. Results from a 5-point Likert scale with 4 representing "substantial progress" and 5 representing "exceptional progress.

\begin{tabular}{lccc}
\hline Learning Objectives & S18 & F18 & S19 \\
\hline Basic Understanding & 4.0 & 4.3 & 4.4 \\
Application & 4.1 & 4.1 & 4.4 \\
Develop Skills & 4.2 & 4.3 & 4.3 \\
Numerical Methods & 4.5 & 4.5 & 4.4 \\
\hline
\end{tabular}

\subsection{Student Feedback}

At the end of every course, students were asked to evaluate the course with the same set of questions. The average response rate was $78.85 \%(81.25 \%, 87.5 \%$, and $70.0 \%$ successively), suggesting a fair representation of the classes as a whole.

The following relevant objectives of the course were self-evaluated by the students on a 5-point Likert scale for the degree of progress: None apparent (1), Slight (2), Moderate (3), Substantial (4), and Exceptional (5). Notably, in each cohort, for each of the four objectives, the average rating was "substantial progress" or higher, showing consistent progress across classes (Table 4).

(1) Basic Understanding: Gaining a basic understanding of the subject (e.g., factual knowledge, methods, principles, generalizations, theories)

(2) Application: Learning to apply course material (to improve thinking, problem solving, and decisions)

(3) Develop skills: Developing specific skills, competencies, and points of view needed by professionals in the field most closely related to this course

(4) Numerical methods: Learning appropriate methods for collecting, analyzing, and interpreting numerical information

Students were asked to provide open-ended comments about the multi-language nature of the course. The questions were phrased to elicit their preference for a single-language course, or if they preferred the ability to choose their own language and observe materials in alternate languages as well. Notably, of the 36 anonymous 
Table 5: Student comments referencing language instruction preferences. Free-form comments were given in response to questions regarding the multiple-language nature of the course

\begin{tabular}{lccc}
\hline Language instruction preference & S18 & F18 & S19 \\
\hline Multiple Language & 10 & 9 & 8 \\
Single Language & 1 & 1 & 1 \\
Ambiguous & 1 & 2 & 3 \\
\hline \% for multiple languages & $83 \%$ & $75 \%$ & $67 \%$ \\
\hline
\end{tabular}

Table 6: Performance for groups of students. This includes grouping by year, sex, and major. Mean performance for year was weighted by fraction of the individual's listed majors in that group.

\begin{tabular}{|c|c|c|c|}
\hline Population & Mean & Std Err & $\mathrm{N}$ \\
\hline \multicolumn{4}{|c|}{ Major } \\
\hline Neuroscience & 84 & 4.3 & 14.53 \\
\hline Psychology & 81 & 6.8 & 6.16 \\
\hline CS/Math/Stat & 86 & 6.2 & 10.16 \\
\hline Business/Economics & 92 & 4.3 & 4 \\
\hline Misc & 84 & 3.4 & 17.14 \\
\hline \multicolumn{4}{|c|}{ Year } \\
\hline Freshmen & 94 & 2.4 & 6 \\
\hline Sophomore & 89 & 3.6 & 9 \\
\hline Junior & 75 & 6.1 & 18 \\
\hline Senior & 94 & 2.4 & 19 \\
\hline \multicolumn{4}{|c|}{ Gender } \\
\hline Female & 87 & 3.3 & 33 \\
\hline Male & 85 & 4.6 & 19 \\
\hline Total & 86 & 2.7 & 52 \\
\hline
\end{tabular}

comments provided, $75 \%$ preferred having instruction available in multiple languages (Table 5).

\subsection{Student Performance}

Performance in the course was assessed by cumulative points acquired throughout the course. The tutorials, assignments, and general structure of the course did not vary substantially across semesters however the quizzes and exams were appropriately updated to discourage use of information from previous semesters. Table 6 indicates the performance among various groups of students. No substantial differences are indicated among groups based on major or gender. The U-shape performance for year (high for freshmen and sophomores, low for juniors, and high for seniors) is influenced by an outlier effect in which the only three students who failed the course were juniors.

\section{CONCLUSION}

The success of this unique course design was consistently observed, so we provide details and resources online for others to benefit from our materials and experiences. The students have consistently rated their progress for each learning objective between substantial or exceptional progress for all three cohorts for each of the four objectives shown. Although resource limitations suggested selecting a single language, this course design included substantial support for multi-language instruction, which was preferred by $75 \%$ of the students. Because of this feedback, the role of this course, originally designed to meet the needs of science undergraduates as a terminal computing course, was expanded to be a required course in a newly designed undergraduate data science major. The use of three languages simultaneously required a design using languageagnostic assignments, which reinforced the role of the course as application-focused rather that tool-focused. We encourage those creating curricula for beginning data science students or science students benefiting from a single computing course to consider this format. And for instructors with limited means to teach such a course in three languages simultaneously, we have made the resources freely available online.

\section{ACKNOWLEDGMENTS}

We would like to thank the following students for their assistance in assembling the materials for the course across the three languages: Emily Phillips (Python), Ian Cowen (R), Abdullah Mazher and Matthew Goldsmith (MATLAB). Ronald Greenberg is supported in part by National Science Foundation grant CNS-1738691. Mark Albert is supported in part by National Science Foundation grant CNS-2051062.

\section{REFERENCES}

[1] ACM Data Science Task Force. 2021. Computing Competencies for Undergraduate Data Science Curricula. https://dstf.acm.org/DSTF_Final_Report.pdf.

[2] Joel C. Adams. 2020. Creating a Balanced Data Science Program. In Proceedings of the 51st SIGCSE Technical Symposium on Computer Science Education. Association for Computing Machinery, 185-191. http://doi.org/10.1145/3328778.3366800.

[3] Jonathan D. Blake. 2011. Language Considerations in the First Year CS Curriculum. fournal of Computing Sciences in Colleges 26, 6 (June 2011), 124-129.

[4] Thomas C. Bressoud and Gavin Thomas. 2019. A Novel Course in Data Systems with Minimal Prerequisites. In Proceedings of the 50th SIGCSE Technical Symposium on Computer Science Education. Association for Computing Machinery, 15-21. http://doi.org/10.1145/3287324.3287425.

[5] Onyeka Ezenwoye. 2018. What Language? - The Choice of an Introductory Programming Language. In Proceedings of the 2018 IEEE Frontiers in Education Conference (FIE). 1-8. https://doi.org/10.1109/FIE.2018.8658592

[6] Yolanda Gil. 2014. Teaching Parallelism Without Programming: A Data Science Curriculum for Non-CS Students. In EduHPC '14: Proceedings of the Workshop on Education for High-Performance Computing. 42-48. https://doi.org/10.1109/ EduHPC.2014.12.

[7] Yolanda Gil. 2016. Teaching Big Data Analytics Skills with Intelligent Workflow Systems. In AAAI'16: Proceedings of the Thirtieth AAAI Conference on Artificial Intelligence. 4081-4088. https://dl.acm.org/doi/10.5555/3016387.3016485.

[8] glassdoor. 2021. 50 Best Jobs in America for 2021. https://www.glassdoor.com/ List/Best-Jobs-in-America-LST_KQ0,20.htm accessed April 9, 2021.

[9] Shriram Krishnamurthi and Kathi Fisler. 2020. Data-Centricity: A Challenge and Opportunity for Computing Education. Commun. ACM 63, 8 (Aug. 2020), 24-26. https://doi.org/10.1145/3408056.

[10] Andrea De Mauro, Marco Greco, Michele Grimaldi, and Giacomo Nobili. 2016. Beyond Data Scientists: a Review of Big Data Skills and Job Families.. In Proceedings of International Forum on Knowledge Asset Dynamics (11th IFKAD). 1844-1857.

[11] John T. Minor and Laxmi P. Gewali. 2004. Pedagogical Issues in Programming Languages. In International Conference on Information Technology: Coding and Computing, Vol. 1. IEEE Computer Society, 562-565. https://doi.org/10.1109/ ITCC.2004.1286523

[12] Kumaresh Pattabiraman. 2019. LinkedIn's Most Promising Jobs of 2019. Linkedin Official Blog. https://blog.linkedin.com/2019/january/10/linkedinsmost-promising-jobs-of-2019 accessed April 9, 2021.

[13] Foster Provost and Tom Fawcett. 2013. Data Science and its Relationship to Big Data and Data-Driven Decision Making. Big Data 1, 1 (Feb. 2013), 51-59. https://doi.org/10.1089/big.2013.1508 
[14] Bina Ramamurthy. 2016. A Practical and Sustainable Model for Learning and Teaching Data Science. In Proceedings of the 47th SIGCSE Technical Symposium on Computer Science Education. Association for Computing Machinery, 169-174. https://doi.org/10.1145/2839509.2844603.

[15] Stephanie Rosenthal and Tingting (Rachel) Chung. 2020. A Data Science Major: Building Skills and Confidence. In Proceedings of the 51st SIGCSE Technical Symposium on Computer Science Education. Association for Computing Machinery, 178-184. https://doi.org/10.1145/3328778.3366791.

[16] Seref Sagiroglu and Duygu Sinanc. 2013. Big data: A review. In 2013 International Conference on Collaboration Technologies and Systems (CTS). 42-47. https://doi.
org/10.1109/CTS.2013.6567202

[17] Lambda School. 2020. The Commons. https://lambdaschool.com/the-commons/ data-science-job-growth-in-2021-and-beyond.

[18] David G. Sullivan. 2013. A data-centric introduction to computer science for non-majors. In Proceedings of the 44th SIGCSE Technical Symposium on Computer Science Education. Association for Computing Machinery, 71-76. https://doi.org/ $10.1145 / 2445196.2445222$

[19] TIOBE Software BV. 2019. TIOBE Index for August 2019. https://www.tiobe. com/tiobe-index accessed August 30, 2019. 